\title{
A FINAL YEAR UNDERGRADUATE DIGITAL CONTROL LABORATORY ASSIGNMENT
}

\author{
J.V. RINGWOOD, C. MCCORKELL and J. WHELAN
}

School of Electronic Engineering, Dublin City University, Glasnevin, Dublin 9, Ireland

\begin{abstract}
This paper describes a positive experience with a digital control assigument used in the BEng in Electronic Engineering course at Dublin City University (DCU). The objective of the assignment is to give the student considerable freedom in tackling a 'real-world' problem, involving modelling, control system design and inplementation. Support is provided through documentation, a standand hardware/software platfom, control system CAD tools and tutorials. The rig used is a fully instrumented coupled tanks alparatus. Completion of the assignment gives students a sense of confidence in tackling other practial, unknown problems, and gives them the sense of being thue 'real-world' engineers.
\end{abstract}

\section{INTRODUCTION}

The BEng in Electronic Engineering at DCU is a four year programme, with optional subjects available only in the final $\left(4^{\text {th }}\right)$ year. The option subjects are allocated two hours per week of lecture time for 26 weeks. Two option subjects are taken and are worth approximately $11 \%$ of the linal degree mark each. The Digital Control Option (DCO) is a relatively popular option, normally attracting about $75 \%$ of the total class number. The laboratory assignment described in this paper is a compulsory coursework module associated with the Digital Control Option, counting for $25 \%$ of the linal subject mark.

The students are exposed to a subject entitled System Dynamics at levels I and II in second and third year respectively. Level I of this subject covers such areas as system modelling, linear systems theory and an introduction to feedback and frequency response. At level II, the concentration is on signals and systems with supplementary material such as properties of feedback systems, frequeny response based stability determination and system identification. In final year, the compulsory course Control Core, containing lectures and assignments in CAD-based control, is taken.

When embarking on the DCO assignment, students therefore have reasonable familiatity with modelling, system identification and CAD tools as well as specific knowledge of the Coupled Tanks Apparatus (CTA) which forms the basis for System Dynamics I and II labonatory experiments. The outstanding knowlenge required consists mainly of analytical examination of digital control systems. discretization techniques and formal control design methods. Expertise required to deal with the electronic hardware aspects of the project are adequately covered in other courses in the BEng programme.

Support for the assignment is given in the form of presentations, tutorials and question/answer sessions (approximately 5-7 hours in total). These hours are included in the 26 hours mentioned above for the DCO. An assignment document is given to all students which indicates the overall structure of the assignment, shows the individual tasks involved and gives some helpful hints. The CTA Manual (Wellstend (1981)) is available to all students.

\section{ASSIGNMENT HARDWARE / SOFTWARE}

An overview of the hardware used in the assignment is shown in Fig.1. For description purposes, the hardware may be divided into a number of interconnected blocks.

\subsection{Coupled Tanks Rig}

The rig itself is manufactured by TecQuipment in the U.K. (Wellstead (1981)) and consists of two equal sized tanks, both square in plan, which are connected by a number of holes. Selective blocking of these holes determines the order of the system and also plays a part in determining the time constants of the system. There is a variable-position tap on the outlet of tank 2, which may be used to control the water throughput and determines the equilibrium heights in both tanks for a given input flowrate. 


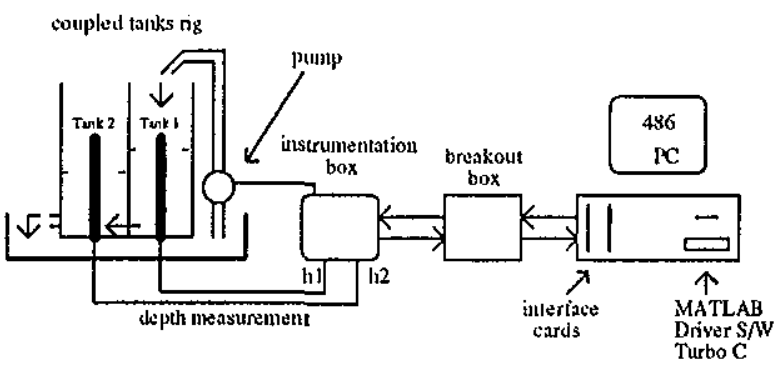

The rig comes complete with depth sensors, but the original resistance-based sensors were replaced with more consistant pressure sensitive devices. The input device is a voltage-fed pump. TecQuipment supply an instrumentation box which provides an electrical interface for the rig. This accepts an input voltage (for the pump) and provides tank height measurement in volts.

This rig is particularly suitable for the assignment due to the following charncteristics:

- The equations which describe the water flow across the various orifices are nonlinear, representing the majority of real-life systems.

- All the system variables are observable with the eye and a conceptual understanding of the system is easily attainable.

- The rig is a pilot-scale representation of problems in the chemical process industries, such as controlling fluid levels in stornge tanks, chemical blending and reaction vessels. It is also representative of problems associated with the distribution and planning of water resources.

- The system is relatively slow, allowing easy observation of dynamical effects in the system, and not putting stringent recquirements on the computing equipment.

- Both states of a linearised state-space description are measurable, providing the statespace basis is chosen appropriately.

- The linearised system is second order, allowing time response specs. to be determined from root locus.

- Real disturbances (dumping water in one or both tanks) are easily generated.

\subsection{Interface Electronics}

The interface electronics comprise that part of the electronics which connects the software variables in the $\mathrm{PC}$ to the electrical variables on the instrumentation box. The operations performed by these electronics include scaling, buffering, $A / D$ conversion and D/A conversion. The $A / D$ board also assists with interrupt generation for synchronization with the sampling period. DAS8 and DAC02 conversions cards from Metrabyte. U.K. are used for $\mathrm{A} / \mathrm{D}$ and $\mathrm{D} / \mathrm{A}$ conversion, respectively. The DAS8 provides 8 multiplexed (single-sided) analog inputs with 8-bit precision, while the $\mathrm{DACO} 2$ has two analog outputs, supplied with 8-bit precision.

\section{3 $\mathrm{PC}$ and Software}

The $\mathrm{PC}$ connected to the rig is a $33 \mathrm{MHz} 486$ IBM compatible. It is networked, allowing easy access to the full suite of deparmental CAD software, but also containing a considerable amount of local hard disk storage for program development. The PC contains, or provides access to, the following software utilities:

- A 'skeleton' program which provides a template for program development. This program presents a convenient interface to the user providing a set of callable functions for sampling period control and reading and writing from/to the $A / D$ and $D / A$ converters. The controller equations (in $\mathrm{C}$ code) may be simply placed in the appropriate section of the template program.

- A Turbo-C environment for controller or logging program compilation.

- MATLAB/SIMULINK for system identification, model validation, control system design and appraisal and simulation.

In addition, a word processing package which facilitates the importing of graphs from MATLAB is available on this and other machines.

\section{ASSIGNMENT STRUCTURE}

The objective of the assignment is to familiarise the student with the practice of designing and implementing digital controllers on 'real-life' processes and to provide exposure to commercial control systems CAD software. To these ends, the assignment contains the following sections:

\subsection{Physical Modelling}

This section involves the determination, with the assistance of the manual, of a set of linearised equations which describe the dynamical characteristics of the system. Examples of the basic relations which govern the system (Wellstead (1981))are:

and

$$
Q_{i}-Q_{1}=A \frac{d H}{d t}
$$

$$
Q_{1}=C_{a 1} a_{1} \sqrt{2 g\left(H_{1}-H_{2}\right)}
$$

describing the rate of change of height in tank 1 and the flow from tank 1 to tank 2. Such equations are 
written for both tanks, expanded in a Taylor series and linearised by retention of the linear terms only. The basis upon which such a linearised model is valid must be clearly understood by the students. The form of the final linearized model is:

$$
\left[\begin{array}{l}
\dot{h}_{1} \\
\dot{h}_{2}
\end{array}\right]=\left[\begin{array}{cc}
\frac{-k_{1}}{A} & \frac{k_{1}}{A} \\
\frac{k_{1}}{A} & -\frac{\left(k_{1}+k_{2}\right)}{A}
\end{array}\right]\left[\begin{array}{l}
h_{1} \\
h_{2}
\end{array}\right]+\left[\begin{array}{c}
\frac{1}{A} \\
0
\end{array}\right] q_{i}
$$

where

$$
k_{1}=\frac{C_{d t_{1}} a_{1} \sqrt{2 g}}{2 \sqrt{H_{1}-H_{2}}} \quad, \quad k_{2}=\frac{C_{d 2} a_{2} \sqrt{2 g}}{2 \sqrt{H_{2}-H_{3}}}
$$

$A$ is the cross-sectional area of one tank.

$\mathrm{C}_{\mathrm{d} 1}, \mathrm{C}_{\mathrm{d} 2}$ denote the orifice discharge cocfficients, $a_{1}, a_{2}$ are the orifice cross-sectional areas,

$\mathrm{H}_{1}, \mathrm{H}_{2}$ are the equilibrium heights in tanks 1 and 2 and $\mathrm{H}_{3}$ is the drain tap height.

The above model shows the linearised relationship between the deviation in tank heights from equilibrium and the deviation input flowrate. To complete the model, the students inust determine values for the model parameters and add on the effects of the pump and depth sensors. This is accomplished using a variety of measurements, static tests and specification data. The final steps in this stage are the selection of a suitable sampling period for the system and the discretization of the system using the zero order hold equivalent (Franklin et al (1990)).

\subsection{Data Logging}

This stage involves the construction and execution of a data logging experiment on the CTA in order to provide data for the following system identification exercise. The template $\mathrm{C}$ program (sce Section 2.3) is available together with a function to generate a PRBS. However, the students are responsible for the specification of the duration of the experiment, sampling period, PRBS minimum swilching interval and amplitude. A complicating factor is the drift in the pump output due to an unregulated PWM driver. This manifests itself particularly over longer durations (e.g. $>1$ hour). Approximately 2000 data points are collected, sufficient for identification in the presence of noise and model validation. Scaling of input and output signals (using potential divider networks) to ensure optimal use of the $A / D$ and $D / A$ ranges is left in the hands of the student.

\subsection{System Identification}

The logged data is now prepared for identification. Both input and output (height in tank 2) signals are detrended i.e. d.c. and first order components are removed. This removes the steady-state heights, concentrating on the deviation variables and also eliminates the effect of drift due to the pump PWM. Half of the recorded points are used for identification and half for validation.

The system identification toolbox (Ljung (1987)) provides a number of user-friendy tools for system identification. These include the loss function examination for a variety of model structures (orders and delay), automatic model structure determination using Akaike's information theoretic criterion (AIC) and parameter identification using a variety of recursive and block identification techniques. For the assignment, three approaches to model order determination can be made:

- Examination of the physical model structure in Section 3.1,

- Examination of the loss function for various model structures, and

- Adoption of the result from applying AIC.

Having justified the choice of model structure, the parameters are identified using a least-squares method.

\subsection{Model Validation}

Tests are now performed to establish the accuracy of the models determined from Sections 3.1 and 3.3. Using the validation section of the PRBS input data used in Section 3.2, the response of the physical and identified models is compared to that from the actual CTA. In addition step tests are compared and discrepancies in transient and d.c. response investigated. If the models produce reasonably acceptable responses, differences are accounted for and a model (either physical or identified) is chosen for control system design and simulation work. Failure to produce an acceptable model may mean recourse to $3.1,3.2$ or 3.3 .

\subsection{Control System Design}

Three control design methods are investigated:

- Three term or PID control,

- Output feedback pole placement, and

- Optimal state feedback.

These three are chosen to demonstrate a subset of the variety of approaches possible. The PID design demonstrates the application of an intuitive, nonmodel-based technique. The model-based output feedback pole placement design (Astrom and 
Wittenmark (1983)) gives exposure to the selection of desired closed-loop characteristics. while the optimal design (Kuo (1980)) shows a state feedback controller which allows control signal weighting.

The desired response specifications are selected in relation to the open loop model and the range of control signals available. In the case of the PID, Ziegler-Nicholls rules are used to determine initial settings on the controller parameters and these are then refined using simulation until a satisfactory reponse is achieved. For the pole-placement design, the response spec. is translated into a set of desired closed loop poles and zeros and a suitable cost function specified for the optimal design. Simulation trials confirm the correct operation of all three controllers and allow iterations on the design specs. Responses to both setpoint changes and disturbances are examined.

\subsection{Control System Implementation}

The designs from Section 3.5 are now implemented in $\mathrm{C}$ code on the PC. Due to familiarity with the hardware from the data logging exercise, this does not present any particular difficulty but students must include some practical additions such as control signal limiting and noise filtering and antiintegral windup for the PID controller especially. Responses to both setpoint changes and disturbances are recorded and differences with the simulated responses accounted for, where possible.

\section{EVALUATION AND ASSESSMENT}

Assignment work and reporting is performed in groups of two. An interim report is prepared at the end of the model validation stage, which is worth $10 \%$ of the DCO mark. This report is corrected and returned to the students to provide feedback on the quality of their work and their expertise in reporting it. A final report, documenting the control design and implementation work is submitted after all the implementation work is complete.

In addition to the assessment of the students, an assessement of the assignment and the level of help given by the students is also performed. This provides useful feedback and allows the refinement of the assignment for future years. The lorm used contains both multiple choice answer format and areas for general comments. Responses were made to the following statements:

Q1: Overall, the assignment has been a positive experience.
Q2: I would now have confience in tackling another practical control design.

Q3: The assignment has heightened my interest in control systems.

Q4: The assignment has given me good insight into the lecture material.

The key to responses is:

1-Strongly disagree , 2-Disagree , 3 - Unsure 4-Agree , 5 - Strongly Agree

The following table documents some of the reponses:

\begin{tabular}{|l|l|l|l|l|l|}
\hline Ques. & 1's & 2's & 3 's & 4 's & 5 's \\
\hline & & & & & \\
\hline 1 & 0 & 2 & 2 & 14 & 5 \\
\hline 2 & 0 & 1 & 8 & 13 & 1 \\
\hline 3 & 0 & 2 & 7 & 11 & 3 \\
\hline 4 & 0 & 1 & 0 & 15 & 7 \\
\hline
\end{tabular}

Under general comments, students most enjoyed designing and simulating the controllers in MATLAB/SIMULINK and seeing their controllers working with the actual coupled tanks apparatus. Most problems and negative comments stemmed from high equipment useage at certain stages of the assignment, restricting access.

\section{CONCLUSIONS}

The assignment froms a very important component of the Digital Control Option at DCU. It provides some motivation for the acceptance of the lecture material and ensures that their control systems expertise is not restricted to the purely theoretical world. Although many students find it time consuming, most feel that the experience is worth the effort.

\section{REFERENCES}

Astrom, K.J. and B. Wittenmark (1983). Computer Controlled Systems - Theory and Design, Prentice-Hall.

Franklin, G.F., J.D. Powell and M.L. Workman (1990) Digital Control of Dynamic Systems, Addison-Wesley.

Kuo, B.C. (1980) Digital Control Systems, Holt, Rinehart and Winston.

Ljung, L. (1987) System Identification - Theory for the User, Prentice-Hall.

Wellstead P.E. (1981) CES - Coupled Tanks Apparatus, TecQuipment Ltd. 